\title{
Elements of automated survey of pavements and a 3D methodology
}

\author{
Kelvin C.P. WANG \\ Civil Engineering, University of Arkansas, Fayetteville AR 72701, USA
}

\begin{abstract}
Sound transportation infrastructure is critical for economic development and sustainability. Pavement condition is a primary concern among agencies of the roadway infrastructure. Automation has become possible in recent years on collecting data and producing results for certain aspects of pavement performance, while challenges remain in several other categories, such as automated cracking survey. This paper reviews the technological advances on automated survey of pavements, and discusses the most recent breakthroughs by the team led by the author in using 3D laser imaging for capturing $1 \mathrm{~mm}$ surface images of pavements.
\end{abstract}

Key words: pavements; automated survey; 3D methodology; surface images

(C) 2011 JMT. All rights reserved.

\section{Introduction}

$\mathrm{L}$ arge infrastructures are usually constructed with materials that exhibit distresses after construction due to various loading, environmental conditions, and aging. The large infrastructures include pavements, chimneys of nuclear power plants, skyscrapers, pipelines, and rail tracks. The distresses are presented in the form of surface cracking in many situations. Successful automation of surface distress survey would reduce the overall cost of performing distress surveys and provide more objective and standardized results for rehabilitation management.

For the inspection of the surface distress of highway pavements, the most widely used method to conduct such surveys is still based on human observation. This approach is extremely labor-intensive, prone to errors and poses hazards. An ideal automated distress detection and recognition system should find all types of cracking and any other surface distresses of any size, at any collection speed, and under any weather conditions. The automated device should be affordable and easy to operate. In recent decades, technological innovations in hardware and imaging recognition techniques have provided opportunities to explore new approaches to automating distress surveys in a cost-effective way. However, despite the performance improvements of newer generation equipment over the older systems, serious

Received Nov. 26, 2010; revision accepted Mar. 18, 2011 *Corresponding author. E-mail: kcw@uark.edu doi: 10.3969/j.issn.2095-087X.2011.01.008 problems still remain in the areas of implementation costs, processing speed, and accuracy.

The goal of this paper is to review design needs of automated systems for the survey of highway pavement surface distress. A particular emphasis of the paper is on using three-dimensional (3D) laser imaging principle for pavement surface, which is capable of capturing true pavement $3 \mathrm{D}$ characteristics at $1 \mathrm{~mm}$ resolution in transverse, longitudinal, and vertical directions. This new technique may provide a platform to re-construct a virtual model at $1 \mathrm{~mm}$ resolution with spatial precision of a pavement surface, of either a roadway or a rail track.

\section{Technical bases and difficulties}

Fig. 1 illustrates the basic system concept of an automated distress survey system, consisting of data acquisition, data storage, and data display and processing subsystems, including a database system for archiving and retrieving the collected and processed data [1].

\subsection{Sensor techniques in data acquisition}

Collecting data on infrastructures in any automated fashion is normally based on man-made sensors. The following factors need to be considered in the use of sensor techniques for the acquisition of pavement surface distress data:

(1) Type of sensor technique to acquire information on pavement surface.

(2) Two dimensional or three dimensional data. 
(3) Severity of distresses, such as sizes of cracks that

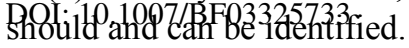

Mendelsohn [2] and others listed possible uses of pavement noises and profiling information to relate to the detection of distresses. Up to today, collection of surface distress data through visual examination is still widely used to find and classify cracks, either through human examination or a man-made vision system. Image data can be collected with various means, such as analog or digital, two-dimensional (2D) camera system, or three-dimensional (3D) laser imaging based acquisition system. In recent years, a common data collection method is to use digital capturing devices, such as a digital camera. The image data in digital format is then transferred to computing devices without the traditional digitizing process for analog based devices, which are no longer used.

Line-scan cameras scan one line at a time, versus area-scan cameras that scan a two-dimensional area at a single pass. Nearly all consumer and cinematic based cameras are area-scan based. Line-scan cameras are mostly used in industrial environment. After decades of relying on area-scan cameras for collecting pavement images, line-scan cameras in recent years became a standard in collecting 2D pavement images. An advantage for using line-scan camera is the ability to illuminate the pavement surface with a line laser. The resulting images are normally of high quality without influencing of sun-light or shadow. With laser illumination, a highly focused narrow laser beam scans a surface in the lateral direction of the movement. The reflections of the laser beam on the surface are then collected with a linescan camera and formulated as a line when one lateral scan is completed. The collected lines are then compiled into a two-dimensional surface.

There is no consensus on what the smallest size of cracks detected should be for engineering purposes. However, if a system can detect a crack with the width of $1 \mathrm{~mm}$, it would be considered adequate. There was a major concern about storage of pavement images several years ago. Two-dimensional images of 4-meter wide pavement at $1 \mathrm{~mm}$ resolution can be archived at the storage consumption of $1 \mathrm{~GB} / \mathrm{s}$, which is no longer a constraining factor.

\subsection{Data processing and difficulties of automation}

Much research and development have been aimed at the computerized processing of the 2D pavement images in order to augment the computer's power with some "human-like" visual sensing capability. This technology, often termed computer vision or machine vision, is related to the second subsystem of image interpretation

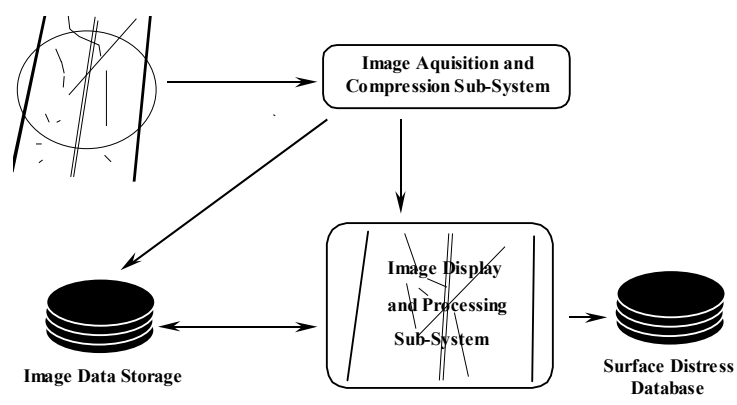

Fig. 1 System concept in the pavement surface distress survey

of collected pavement surface data, or data processing in Fig. 1.

Humans can detect and classify pavement surface distress with ease, but at very slow speed. Computer vision systems distinguish cracks through identifying disturbances in the brightness range of the surrounding texture and must be designed to seek connected regions through mathematical algorithms. Even if all the imaging processing handled by human brain were understood, it was possible to implement that knowledge into a computer to make its performance comparable to human's, the amount of processing power required is of the order of 1012 billion floating point operations per second (one teraflops) [3]. A workstation at a reasonable cost and augmented with a high-end imaging board can only achieve the order of several billion float point operations per second (gigaflop).

The highway industry would like to apply automated distress survey equipment with real-time processing capability, and acceptable consistency, repeatability, and accuracy. It has been a frustrating period in the past two decades for developers to implement distress survey systems based on these requirements.

(1) Image processing for pavement surface distress survey at any practical speed requires very high performance computing equipment. When a compromise is made in respect of computing performance, data quality, processing speed, or both are affected.

(2) Image processing as a field of study is still evolving. There are many aspects of image processing in human brain that are not understood yet.

(3) In the detection and recognition of pavement surface distress, a particular difficulty is related with the surface texture and foreign objects on the pavement surface, such as oil spoil.

(4) There are no standard indexes to quantitatively define the types, severity, and extent of pavement surface distress. However, efforts are underway to initialize a set of standards [4-6].

(5) It is not necessary to have compatibility of hardware and software with different vendors' systems. 
However, this incompatibility introduces noncomparable.1007/BF survey 325733 different vendors.

In the early 1990s, Texas Department of Transportation and US Federal Highway Administration organized a trial test of existing automated systems. Vendors in highway data collection business were invited to conduct surveys with their automated equipment. There was not much agreement among the results from different vendors and the trial test did not produce a comparison evaluation of the various devices. Smith [7] led a team and conducted a study on the existing survey equipment on pavement surface distress. Four vendors were invited and participated in the study. Most of the four vendors only have capabilities of collecting pavement surface images. The analyses of surface distresses were conducted either manually, or with the assistance of a vision system under manual control.

Since the 1980s, five major efforts that produced working systems with the capability of at or near realtime processing are: the Japanese Komatsu system, the US PCES system, the Swedish PAVUE system, the Swiss CREHOS, and the Illinois Automated Road Inspection System. Descriptions of the efforts are given by Wang [1].

\section{2D based approach using line-scan camera}

\subsection{Principle}

The Time Delayed Integration (TDI) camera is a new type of high-performance line-scan camera used for high speed and relatively low lighting applications. TDI makes use of synchronous motion to take multiple pictures of the same line image and add them up to get an amplified image. The high sensitivity of TDI cameras is due to image integration over multiple stages. For example, compared with regular line-scan camera, a TDI camera can have 96 stages, resulting in 96 times the integration period. The overall sensitivity is improved by a factor of 80 , due to added noise sources in the TDI sensors.

In any line-scan application, the system designer must consider both the resolution across the object's movement (transverse resolution) and the resolution along the path of the object's movement (longitudinal resolution), as demonstrated in Fig. 2. In the design of line-scanning based data vehicle, the transverse resolution is limited only by the number of line pixels in the camera. Longitudinal resolution is a function of the speed of movement, or the data vehicle's speed, and the scan rate of the camera. The inspection with the linescan camera can be $100 \%$ coverage of the surface at one pre-determined resolution at both longitudinal and transverse directions. The speed encoder in the figure generates and sends the speed data to the camera at real-

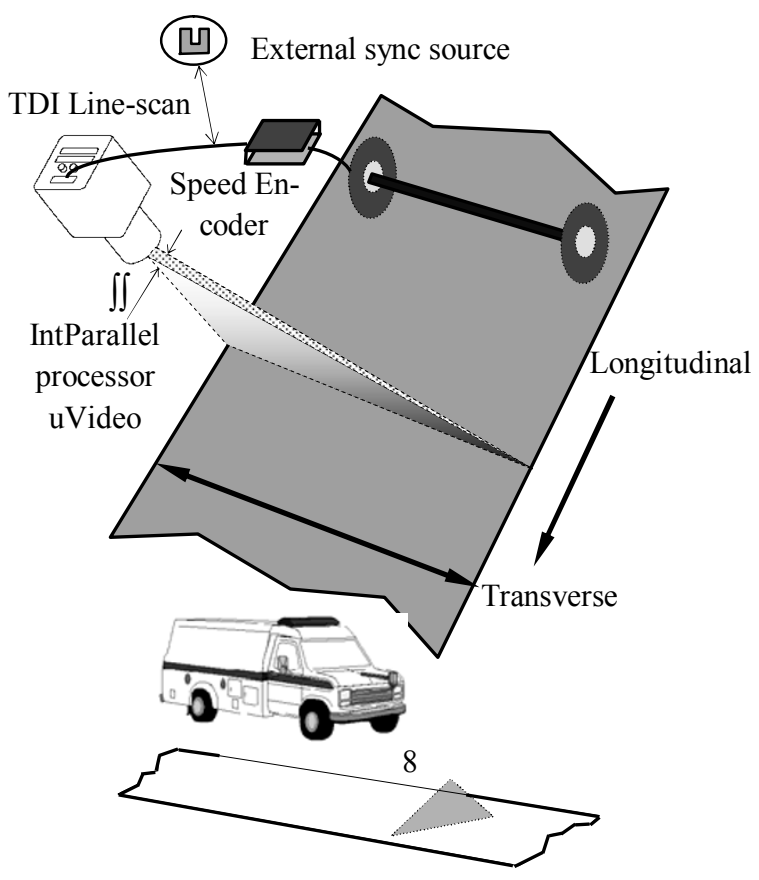

Fig. 2 A line-scan camera based pavement surface inspection vehicle

time. With the speed known and the resolution fixed, scan rate of the camera can be dynamically adjusted to satisfy the requirement of uniform resolution for both transverse and longitudinal directions. The performance of a high-end TDI camera can be obtained at a line rate over $40 \mathrm{kHz}$. The principle to establish a $2 \mathrm{D}$ image of the pavement surface is as follows:

$$
\begin{aligned}
& \text { Pavement surface image }= \\
& \quad \iint f(\text { raw TDI lines }) \mathrm{d} l \mathrm{~d} x,
\end{aligned}
$$

where $l$ is images lines before integration, and $x$ is distance of traveling.

In order to capture clean images with a TDI camera, tight synchronization with the moving vehicle is required. Barbe [8] presents the Modulation Transfer Function (MTF), relating to the effect on image quality by speed variation:

$$
\operatorname{MTF}(T D I)=M \Delta V / V,
$$

where $M$ is the number of stages, and $\Delta V / V$ is ratio of change in velocity to the average velocity during the integration period for the photo-elements of all stages.

Barbe also shows that when MTF is less than 2, there is no effect on image quality. For instance, when $M=96$, $\Delta V / V$ can be as high as $2 \%$. It is possible to control the speed variation of the data vehicle within this range. If the variation is over $2 \%$, for instance, when $\Delta V / V=5 \%$, the number of stages can be reduced accordingly so that 
MTF is always less than 2. Certain TDI cameras may alPow the dynamic adjustment of stage number.

Side-to-side motion can also affect the image uniformity when photo-elements in the same column may capture unnecessary pixels. Sideways velocity must be limited to $1 / 96$ th of the vehicle speed to limit the effect. 96 are the total stage number in the TDI camera. Based on integration needs, the number of stages may be adjusted or selected for certain TDI cameras. The effect of side to side motion on image quality can also be controlled through adjusting the number of stages.

\subsection{System design of line-camera based $2 D$ data collec- tion}

The principle of all systems that inspect moving objects is to achieve stop action within the resolution of the smallest point of interest. New TDI cameras overcome limitations of area-scan, laser scan and regular line-scan cameras with high resolution, a high line rate and a magnitude higher sensitivity to light. Fig. 3 illustrates a system design for an inspection system for pavement surface distress with a TDI camera.

The speed of the data vehicle is monitored in the encoder to determine the synchronization with the camera. The timing control unit generates necessary camera clock signals based on the data from the encoder. Lines from the camera are formatted in the Video Formatting step in the camera interface device. The formatted digital lines are then fed into a parallel processor for image processing. All the interfacing devices and the parallel processor are housed in the host computer. The host computer also controls disk array for the storage of the images and related data sets. In addition, a GPS receiver is connected to the host computer to log location data for the collected images.

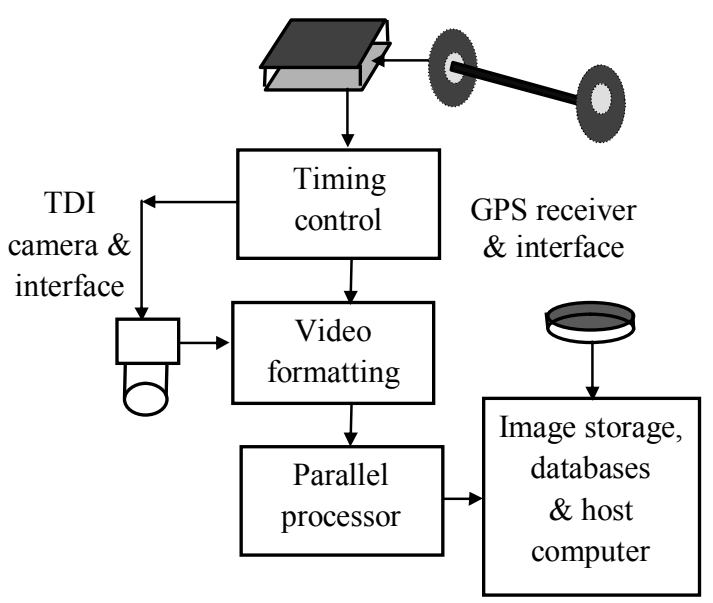

Fig. 3 Elements of TDI based surface inspection system

\section{Basis of 3D data collection technology}

3D surface features of pavements have been studied closely for years for various data analysis needs. However, true 3D surface measurements of pavements obtained for computer analysis at high resolution and at highway speed have not been obtainable for production purposes. Rather, for decades, 2D images have been used by pavement engineers to estimate pavement distress, with less-than-desirable results. It was therefore natural to conclude that in order to have better solutions to pavement surface evaluation, obtaining pavement surface information in its original format, or a 3D representation, at sufficient resolution such as $1 \mathrm{~mm}$, represents a new research frontier. However, this development heavily relies on advancement of sensor and computing technologies due to the need of high resolution, complete coverage of pavement lane, sustained high data rates of acquisition and analysis, and overcoming adverse influences of sun light and ambient light during data acquisition.

There are several techniques to collect 3D surface data. A conventional method is based on the photogrammetric principle, which has been widely used in highway engineering dating to the use of analog film. From 2005 to 2007, the NCHRP IDEA program funded the team to use the photogrammetric principle to establish 3D pavement surfaces in the project NCHRP-88, "Automated Pavement Distress Survey through Stereovision" [8]. The research produced good results. However, the critical limitation of this technique is the lighting requirement for the paired cameras to obtain high fidelity 2D images of the pavement surface. Even today, the illumination of a pavement surface to the required intensity level under direct sunlight for a full-lane area is nearly impossible, which is required for photogrammetric image acquisition in order to have high-quality $2 \mathrm{D}$ visuals to establish common points in the paired images. Fig. 4 illustrates the photogrammetric principle used in the NCHRP IDEA project and the resulting software to match a pair of 2D images with common points to generate a 3D surface model of pavement.

Another technique for 3D surface modeling is Light Detection And Ranging (LIDAR), which was initially used to geo-reference terrain features. In some literature LIDAR is referred to as laser altimetry. A LIDAR system shown in Fig. 5(a) is composed of a laser scanning system, GPS receiver, and an IMU [9]. The laser scan data is collected using a scanning mirror that rotates transverse to the direction of flight or motion. LIDAR signal is not a point but rather is an area beam. The beam is very narrow, but it does get larger as it moves away from the source. 

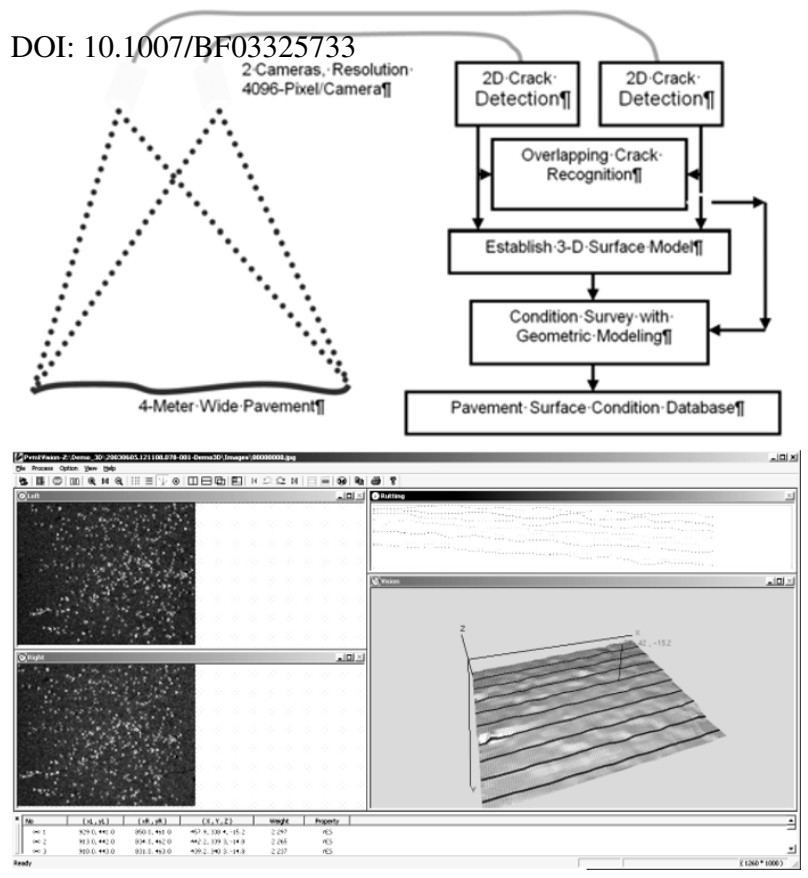

Fig. 4 Basis of stereovision for condition survey and the $3 \mathrm{D}$ reconstruction software [9]

Moreover, the laser beam also becomes distorted, taking on an ellipsoidal shape, as it travels along the scan [10]. Based on LIDAR principle, Fig. 5(b) shows a rotating laser system for pavement survey developed in the 1990's by Phoenix Scientific [11-12]. Devices based on the rotating laser principle received widespread attention and enthusiasm early on. Due to difficulties in making significant improvements to the resolution of the system in the last decade, and the popularity of laser based 2D imaging system introduced several years ago, the usage of the technique based on the rotating laser principle has been limited to niche applications.

Fig. 6 illustrates a 3D laser imaging technique that has been widely used for object inspection on conveyor belts in the recent decade. By illuminating a surface using a line laser and shooting 2D images using an area camera from the side (an angle) targeting at the narrow area of the laser line, the surface variation in the vertical direction can be analyzed by examining the laser line features in the captured 2D picture. When 2D images are captured in a sequence, the laser lines in the sequential 2D pictures can be extracted and combined sequentially to form a digital 3D surface.

This technique has been used by the research team to develop a 3D prototype system for pavement surface data collection to produce $1 \mathrm{~mm}$ resolution $3 \mathrm{D}$ images of pavement surfaces, including the sample 3D images shown in the proposal. It should be noted that the cameras used with this technique are commonly referred to as $3 \mathrm{D}$ cameras, despite the fact that they are specialized 2D cameras that have dedicated processors inside the

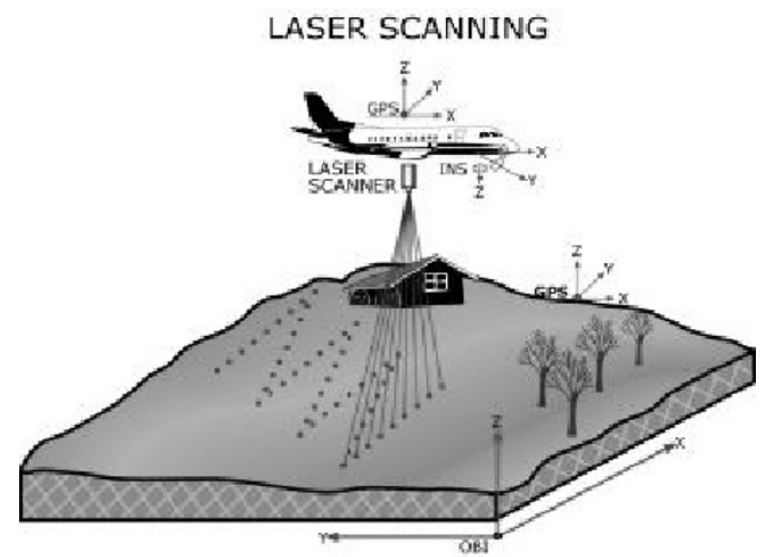

(a)

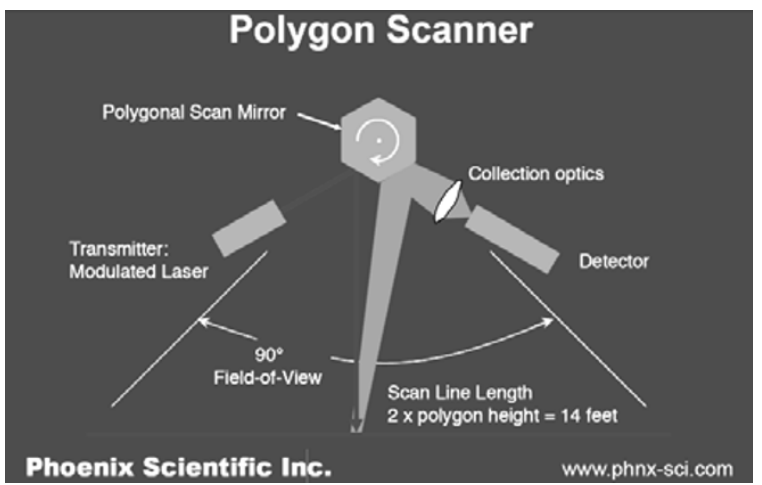

(b)

Fig. 5 LIDAR principle (a) and rotating laser system for pavement survey (b)
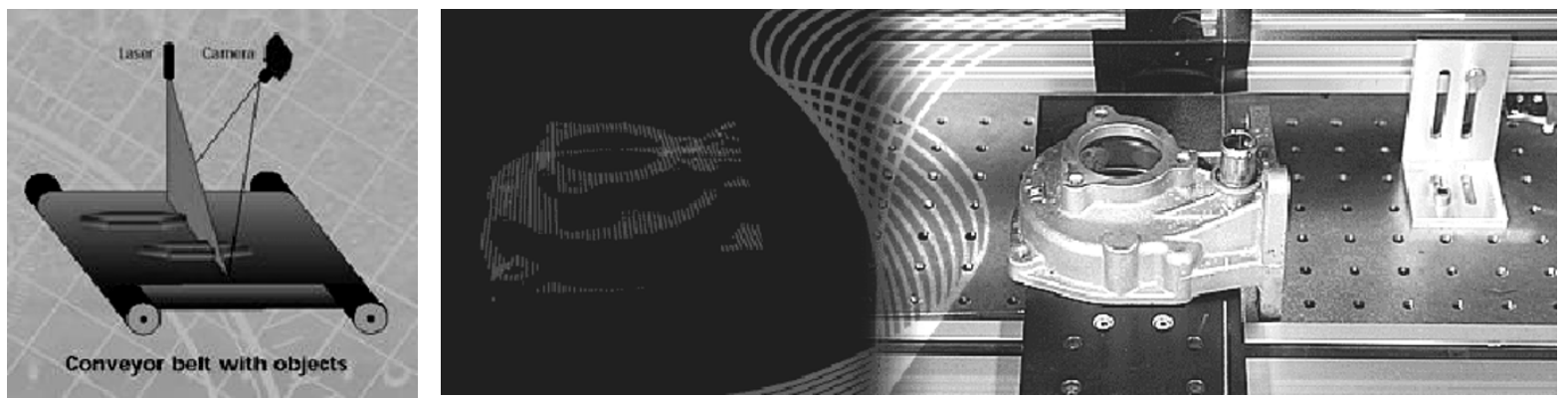

Fig. 6 Conveyer belt (http://www.adept.net.au/news/newsletter/200810-oct/3D_Camera.shtml) 
Domeras 1007/BF03325733 extract lines from the captured 2D images.

3D cameras based the line laser principle can only produce height information at pixel points. Laser intensity information on pavement surface from 3D cameras is generally of poor visual quality. As pavement surface imagery is important supplementary information to the $1 \mathrm{~mm}$ 3D surface models for both purposes of visualization and automation algorithm for distresses, the new research will integrate a $1 \mathrm{~mm}$ resolution 2D laser imaging sub-system, resulting in a new hybrid 2D/3D data acquisition system.

\section{Preliminary results of the $3 D$ laser imag- ing system, PaveVision3D}

The new system collectively called PaveVision3D will have four major capabilities integrated into a single vehicular surveying platform: surface distresses, profiling (transverse for rutting and longitudinal for roughness), macro-texture, and roadway geometry. All data sets in the survey vehicle will be obtained at highway speed and processed in real-time. Software solutions for the four capabilities are among the key contributions of the proposed research that no other research teams have demonstrated with $3 \mathrm{D}$ data to date. It is anticipated that efforts will be made in the research to conduct precision and bias analyses based on various ASTM and AASHTO protocols against widely accepted and certified measurement devices for macro-texture and longitudinal profile, and manual surveys of pavement surface distresses.

The PaveVision3D will be based on a single vehicular platform (Digital Highway Data Vehicle, DHDV) shown in Fig. 7 with entire-lane area coverage for all collected surface data sets. The research tasks include designing and constructing the hybrid 2D/3D laser imaging system with the following unique characteristics:

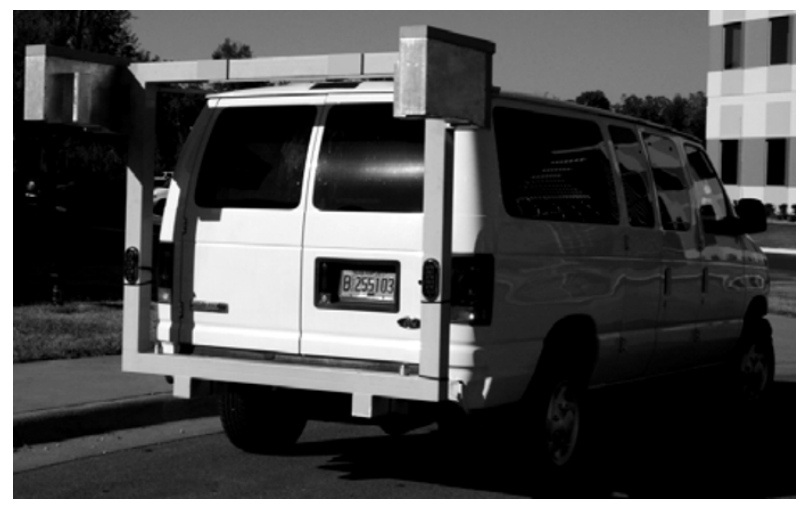

Fig. 7 The prototype DHDV with two 2D/3D hybrid sensor cases of PaveVision3D
(1) Integrated arrays of 3D cameras, lasers, accelerometers, multi-CPU and multi-core computing devices, and differential GPS receivers and one high-precision IMU.

(2) Software algorithms to provide the four automated capabilities: surface distresses, profiling (transverse for rutting and longitudinal for roughness), and macro-texture, and roadway geometry.

(3) 3D data for entire surface of pavement lane at $1 \mathrm{~mm}$ resolution in transverse $(x)$, longitudinal $(y)$, and height $(z)$ directions at the data collection speed of $60 \mathrm{mph}$ (100 kph).

(4) A sub-system to acquire $1 \mathrm{~mm} 2 \mathrm{D}$ laser images of surface imagery. 3D and 2D data streams are synchronized for location accuracy.

Fig. 8 shows the sensor system configuration of PaveVision3D in a DHDV. Each sensor case covers half of a lane and contains two sensor assemblies: 2D laser imaging for $1 \mathrm{~mm}$ visual images, and 3D laser imaging for $1 \mathrm{~mm}$ surface information. Fig. 9 illustrates an actual data set obtained from the prototype DHDV equipped with PaveVision3D hybrid sensors: the gray-scale visual imagery on the left from the 2D laser assembly, and rainbow 3D imagery on the right from the 3D laser assembly. The bar of red line on the 3D surface in Fig. 9 is

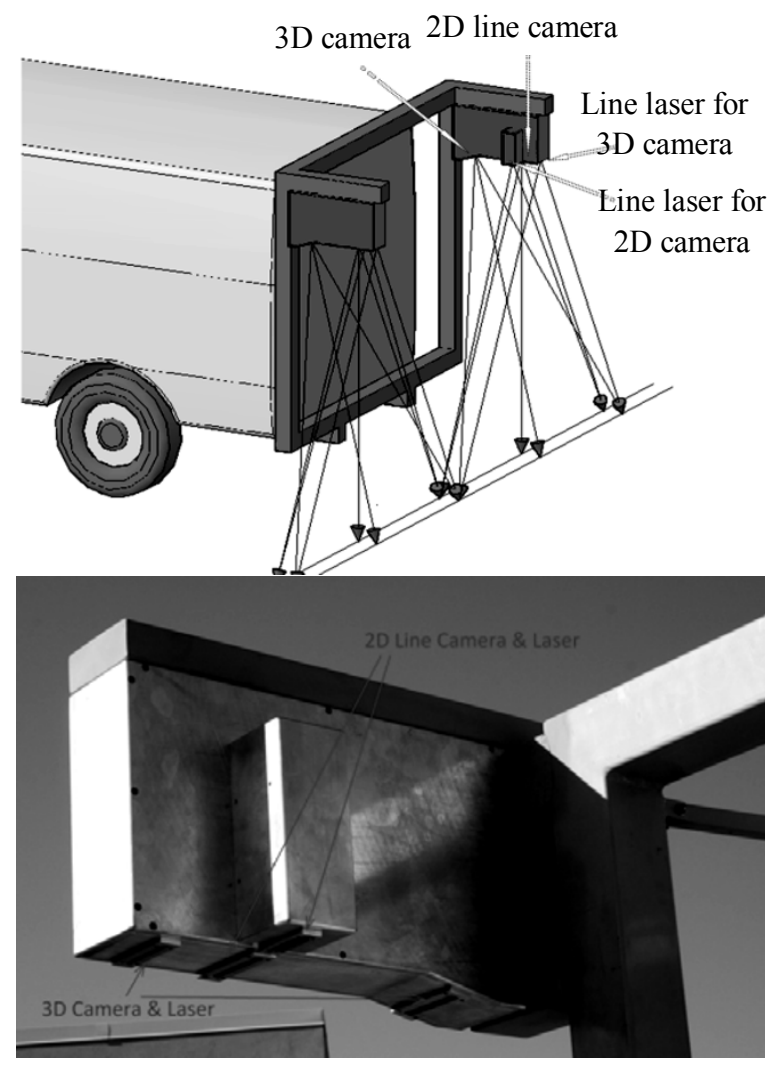

Fig. 8 Sensor system configuration (top) \& a laser imaging sensor (bottom) 
the virtual measurement tool of transverse profile.

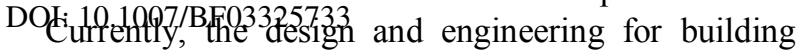
two prototyping sensor cases has been completed shown in Fig. 7 and Fig. 8, including laser assembly, cameras, lenses, filters, trigonometry relationships, and geometric dimensions to construct the cases. 2D line cameras at 2048 pixels are used to capture surface imagery of pavement at $1 \mathrm{~mm}$ resolution in both transverse and longitudinal directions. A 2D line camera covers about half lane or 2-meter wide area. A 3D camera at the resolution of more than 2000 pixels in the transverse direction is used to cover half-lane as well with $3 \mathrm{D}$ profile rate at 6000 per second.
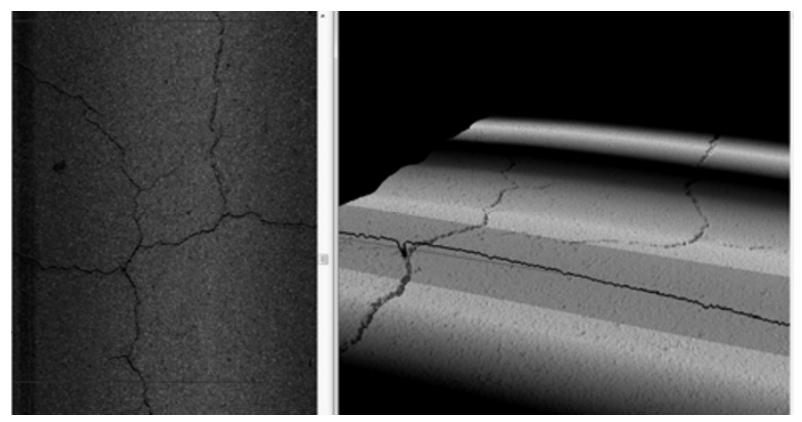

Fig. 9 Sample 2D and 3D data of an actual pavement area

The assembled prototype system can operate during any weather condition as long as pavement surface is dry. Due to the effective use of wavelength filters and line laser emitters in the prototyping system, sun light does not influence data quality for both $2 \mathrm{D}$ imagery and 3D surface data. The prototype system can operate at night or in day time to collect both $2 \mathrm{D}$ and $3 \mathrm{D}$ data at the same quality levels.

\section{Conclusion}

The desired features of the research include a vehicular based operational system that (1) uses integrated remote sensing and space-based technologies and products for monitoring transportation infrastructure conditions; (2) possesses new capabilities for asset management, inspection, and condition assessment for continued serviceability of constructed infrastructure; (3) promotes usable applications which would add value and enhance current transportation business practices adopted by state and local transportation departments. In the last two decades, sensor and computing technologies have improved by orders of magnitude. The 3D technology represents an opportunity to revolutionize the process of data collection and processing. The demonstrated prototype 3D system developed by the research team has the potential to be the basis to provide the industry a total solution for automated pavement surface data collection and processing.

\section{References}

[1] K.C.P. Wang, Design and implementation of automated systems for pavement surface distress survey, ASCE Journal of Infrastructure Systems, 2000, 6(1): 24-32.

[2] D.H. Mendelsohn, Automated Pavement Crack Detection: An Assessment of Leading Technologies, In: Proceedings of the Second North American Conference on Managing Pavements, Vol. 3, Toronto, Canada, 1987: 3.297-2.314.

[3] D. Crevier, Daniel, Computer Vision and Artificial Intelligence, Advanced Imaging, NY: Melville, 1997: 60-63.

[4] W.D. Paterson, Proposal of Universal Cracking Indicator for Pavements, Transportation Research Record, 1994(1455): 69-76.

[5] FHWA, Protocol for Distress (Tentative), by US Federal Highway Administration and TRDF, 1997.

[6] ASSHTO, Standard Practice for Collecting Images of Pavement Surfaces for Distress Detection, AASHTO Designation, 2010: 68-10.

[7] R.E. Smith, T.J. Freeman, O.J. Pendleton, Evaluation of automated pavement distress data collection procedures for local agency pavement management, In: Proc. of 4th International Conference on Managing Pavements, 1998: 269-286.

[8] D. F. Barbe, Time delay and integration image sensors, In: Solid State Imaging, P. G. Jespers, F. van de Wiele, and M. H. White eds. Leyden, Netherlands: Nordhoff, 1976: 659-671.

[9] K.C.P. Wang, Automated Pavement Distress Survey through Stereovision, NCHRP-IDEA Program Project Final Report, Washington, D.C., 2004.

[10] R. Burtch, Lidar Principles and Applications, In: Proceeding of IMAGIN Conference, IMAGIN Conference, Traverse City, MI, USA, April 29-May 1, 2002: 1-12.

[11] B. Herr, Calibration and Operation of Pavement Profile Scanners, RPUG, Lake Tahoe, 2001.

[12] B. Herr, PSI Current Technology Overview, white paper, July 11, 2009.

(Editor: Junsi LAN) 